\title{
Training of pathologists by a system of modules
}

\author{
JOHN STUART AND PHILIP A. GORDON \\ From the Department of Haematology, The Children's Hospital, Ladywood Middleway, Birmingham
}

SYNOPSIS The training requirements of the individual pathology subjects within laboratory medicine? are changing rapidly and there is a trend to earlier specialization. A new training scheme based on a $\vec{\omega}$ system of 10 modules, each of six months' duration, is suggested. Six of the modules comprise obligatory specialist training while the remaining four provide an element of choice both in subject? matter and timing. This allows the advantages of multidisciplinary training to be retained, despite ${ }^{i}$ earlier specialization, since the subjects studied need not necessarily be restricted to the traditionali + pathology disciplines. The system allows $20 \%$ of training time to be devoted to full-time research $\omega_{\tilde{W}}^{\omega}$ and has been designed to be attractive to trainees in general medicine. Modular training provides ${ }_{\circ}^{+}$ an inherently flexible system which can readily be adapted to future developments in pathology and ${ }_{-}$ medicine.

\section{The Existing System}

Laboratory medicine is a rapidly developing subject with a complex training structure which requires regular revision. The Royal College of Pathologists has maintained a flexible attitude to the training of pathologists in the United Kingdom and progressively modifies its examination system as the requirements for training change. The present twostage examination system requires an assessment at the end of two years of general training and again on completion of a further three years of specialist training. A high standard is set in the examinations, and the College's recommendations on training and also its examination system have done much to ensure a uniformly high standard of competence among its members.

\section{Areas of Debate within this System}

MULTIDISCIPLINARY TRAINING

As a consequence of increasing specialization there is debate over the value of the initial two-year period of general training in more than one pathology subject. This period has the advantage of providing a broad background for the pathologist who will subsequently direct a multidisciplinary laboratory or have responsibility for more than one subject during vacation periods. It is also of value to trainees who are uncertain as to which specialty to

Received for publication 18 January, 1974. adopt. For the committed specialist the value is less clear, since the time might be more usefully employed in the specialty itself, and to many this trendo to earlier specialization is logical and inevitable. As period of general or additional training can, however, be of considerable value even to the committed $\frac{\mathrm{Q}}{\mathbb{Q}}$ specialist provided the subjects are not too remote from the chosen specialty. A trainee in clinicali $\overrightarrow{\overrightarrow{0}}$ chemistry, for example, might obtain more benefit 3 from six months spent in a renal or endocrine unit, or in immunology or general medicine, than in $a$. routine microbiology or haematology laboratory. The major problem is the selection of the best type of training for the individual requirements of each 3 . trainee. This is extremely difficult to achieve sinces the nature of training in the individual pathology disciplines differs significantly and since newo trainees may not be aware of their future requirements. If the advantages of a period of generato training are to be retained then it would seem necessary to allow an element of choice in both thes nature and timing of the subjects studied.

\section{CLINICAL V LABORATORY HAEMATOLOGY} The training of a haematologist is even more complex owing to the additional requirement for a clinica component during training. There has been con $\frac{\mathbb{D}}{5}$ siderable debate over the relative merits of a clinicat as opposed to a laboratory training but the division is seen to be an artificial one and a practising haematologist requires to have experience in both fields (Joint Committee on Higher Medical Training, $\varrho$ 
1973). The area of debate has now moved on to determine how this form of training can best be achieved.

\section{ASSESSMENT BY EXAMINATION}

The flexible attitude of the Royal College of Pathologists extends to trainees with a less conventional background and periods spent abroad, in research, and in district general hospitals are encouraged. Exemption from the primary and/or final examinations of the College can be achieved by the submission of published work; exemption from the primary examination may also be granted to a member of a Royal College of Physicians.

Despite this initiative on the part of the Royal College of Pathologists, medical graduates in the United Kingdom remain excessively conditioned by the examination system. Many trainees with the ability to carry out some original research, but not to the extent of gaining examination exemption, consider Membership of the Royal College of Pathologists, and/or Physicians, to be their only major goal. In laboratory medicine, the requirement for a final examination on completion of specialist training, plus a rotational training programme which dictates a change of laboratory every six to 12 months, often compete to the detriment of parttime research projects.

Irrespective of the stimulus provided by heads of departments the present system does not encourage, in the average trainee, the attitude of mind which is likely to produce original work. This would seem to be a serious omission in a subject which is developing as rapidly as laboratory medicine. The difficulty lies in maintaining the advantage of the present training system, which ensures a high standard of competence in the trainee, while encouraging a more positive approach to the educational value of research and developmental work.

\section{Training by a System of Modules}

It is suggested that the training of a pathologist should comprise a series of 10 modules, each of six months' duration. This modular system would allow the trainee to retain an element of choice, within broad limits, in the timing of each module and in its content. It is envisaged that some trainees would select four traditional pathology subjects as modules during the first two years, ie, the conventional multidisciplinary general training, and then progress to six modules of specialist training. Other trainees, more dedicated to specialization at an early stage, might first undergo the six modules of specialist training and then use the final four modules to study additional subjects closely related to their chosen specialty. An example of this system as applied to haematology is described.

\section{Modular Training Scheme in Haematology}

A list of module subjects which would be obligatory for specialist training is given in table I. Each module subject could be studied separately or two subjects, but not more than two, studied simultaneously, eg, clinical and laboratory haematology studied simultaneously to a total of five modules. Specialist training would consist of at least three years (six modules) but additional modules could be taken in any of the subjects if desired.

\begin{tabular}{ll}
\hline Subject & $\begin{array}{l}\text { Minimum Number of Modules } \\
\text { (Each of Six Months' Duration) }\end{array}$ \\
\hline Laboratory haematology & 3 \\
Clinical haematology & 2 \\
Blood transfusion/serology & 1 \\
\hline
\end{tabular}

Table I Obligatory module subjects for specialist training in haematology

Examples of optional module subjects, for general or additional training, are given in table II. These subjects could be studied either before specialist training, or as an interruption of specialist training, or at the end of it. Four modules, comprising at least two subjects, would require to be taken from this section. Each module subject could be studied separately or two subjects, but not more than two, studied simultaneously, eg, a senior house officer appointment held jointly in clinical haematology and general medicine.

\begin{tabular}{ll}
\hline General Training & Additional Training \\
\hline Clinical chemistry & General medicine (postregistration) \\
Haematology & Clinical haematology \\
Histopathology & Laboratory haematology \\
Microbiology & Blood transfusion/serology \\
& Immunology \\
& Virology \\
& Nuclear medicine/isotopes \\
& Radiotherapy/oncology \\
& Genetics/chromosomes \\
& Research (full time) \\
\hline
\end{tabular}

Table II Examples of optional module subjects to be taken as general or additional training ${ }^{1}$

${ }^{1}$ A minimum of four modules, comprising at least two subjects, should be studied.

This list of optional module subjects is deliberately wide-ranging to provide either a broad-based training 
for the general trainee or a range of options for the more academic haematologist. It would be possible to study more than four modules from this section and, in particular, a trainee recruited from general medicine might already have completed more than four modules in that subject. It is recommended, therefore, that not more than two modules in each of the general or additional subjects be credited for examination purposes and that at least two of these modules be taken in a laboratory discipline. The recruit from general medicine could therefore count one year of postregistration clinical experience as two modules but would require to complete two further modules in laboratory medicine (which could consist of research) in addition to six modules of specialist training.

\section{Advantages of Training by Modules}

The modular training scheme has the advantage that it provides multidisciplinary general training for those who want it. A haematologist, however, might prefer to build up additional modules in general medicine, immunology, and research rather than the more traditional subjects of microbiology, histopathology, and clinical chemistry. The system also allows $20 \%$ of training time to be devoted to fulltime research. The scheme has been designed to attract trainees with postregistration experience in general medicine, this will become increasingly important, and will allow a haematologist with scanty postregistration general medical training to return to full-time clinical work for one year (two modules of optional training).

The existing two-stage examination system of the Royal College of Pathologists could be superimposed on a modular training scheme with the examinations held on completion of four and 10 modules respectively. These examinations would be difficult to organize, however, owing to the different backgrounds of individual trainees. The modular concept favours more a system of continuous assessment, based on the successful completion of individual modules in accredited departments, but this raises further difficulties because of the subjective nature of the assessment.

It does seem advisable to have one formal assessment by examination at some stage during the training period and preferably before the end of it. This examination could be based on the subject matter of the six modules required for specialist training and could therefore be taken at any stage following completion of these modules. The remaining four modules of additional training, which require to be undertaken in accredited departments, need only be assessed by the submission of detailed reports from the heads of individual departments. The committed specialist could therefore complete his six obligatory modules within the first three years of training and be free from formal examination thereafter.

\section{Conclusions}

All training schemes have advantages and disadvantages both to trainees and to heads of departments. This proposed modular training scheme in pathology has been based on a number of recent modular courses in non-medical faculties which are proving popular with undergraduates. It is suggested as a form of postgraduate training in an attempt to satisfy the different requirements of the individual pathology disciplines; it also avoids the conflict between multidisciplinary general training as opposed to early specialization.

The flexibility of the system, which allows a year of full-time research, or of training in an accessory subject, to be inserted at any stage of a five-year programme, does increase the complexity of rotational training programmes, Senior registrars are eligible to apply for off-service elective periods, and some rotational training schemes already provide for this, but the demands of service responsibilities may make this difficult to achieve on the larger scale required for the modular system.

The problem of registration of departments as suitable for training in individual module subjects is not a major one since pathology departments already require to be accredited for training. Individual trainees would, however, require guidance as to a suitable choice of module subjects for training in the different pathology disciplines but it would not be difficult to provide illustrative examples.

One benefit claimed by protagonists of modular training schemes is that the trainee becomes motivated at an early stage of his career since he is able to construct a package of subjects which are of direct relevance to his chosen specialty. This concept, linked to a system of continuous assessment to supplement, and in part replace, major examinations and with an optional period of full-time research, would provide a unique and potentially attractive form of postgraduate medical education which could stimulate recruitment to pathology.

\section{Reference}

Joint Committee on Higher Medical Training (1973). Specialis Advisory Committee on Haematology, Recommendations on Training. Royal College of Physicians, London.

\section{$\Rightarrow$}

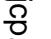
N is $\omega$

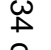
윽 